$\|y(0)\| \geqq a,\left\|y\left(x e^{i \theta}\right)\right\| \geqq u_{\theta}(x)$ on $[0, r(\theta))$, of significance up to the first positive zero of $u_{\theta}$.

\title{
REFERENCES
}

1. K. M. Das, Singularity-free regions for solutions of 2 nd order non-linear differential equations, J. Math. Mech. 13 (1964), 73-84.

2. P. Hartman, Ordinary differential equations, Wiley, New York, 1964.

3. E. L. Ince, Ordinary differential equations, Dover, New York, 1926.

4. V. Lakshmikantham, Upper and lower bounds of the norm of solutions of differ ential equations, Proc. Amer. Math. Soc. 13 (1962), 615-616.

UNIVERSITY OF UTAH AND

Montana State University

\section{ASYMPTOTIC ALMOST PERIODICITY OF SOLUTIONS OF A SYSTEM OF DIFFERENTIAL EQUATIONS}

\section{W. R. UTZ AND PAUL WALTMAN ${ }^{1}$}

The theorems of this paper give sufficient conditions for the asymptotic almost periodicity of bounded solutions of a system of differential equations in the plane. Let $x=\left(x_{1}, x_{2}\right)$ and let

$$
\rho(x, y)=\|x(t)-y(t)\|=\left|x_{1}(t)-y_{1}(t)\right|+\left|x_{2}(t)-y_{2}(t)\right| .
$$

The system to be considered, using this vector agreement, is

$$
x^{\prime}=f(t, x) \text {. }
$$

The theorems that we prove were suggested by a paper of J. S. W. Wong and T. A. Burton [4] who consider the system (1) of the special form

$$
x_{1}^{\prime}=x_{2}, \quad x_{2}^{\prime}=-a(t) f\left(x_{1}\right) g\left(x_{2}\right) .
$$

The proofs of our theorems differ considerably from the proofs of Wong and Burton. An important difference is our use of periodic solutions of a limiting system of (1) to avoid the use of Liapunov functions.

A solution, $x=x(t)$, of (1) valid for all large $t$ is said to be asymptotically almost periodic if there exists a positive real number $T$ such that for every $\epsilon>0$ there corresponds a real number $t(\epsilon)$ for which

Presented to the Society, April 14, 1967; received by the editors August 1, 1966.

1 Research of this author supported by NSF Grant GP-5599. 


$$
\rho(x(t), x(t+T))<\epsilon
$$

for all $t \geqq t(\epsilon)$.

Wong and Burton call this property "almost periodic for $t$ sufficiently large". The nomenclature used in this paper seems more appropriate to the authors even though it has had a prior, and different, use by Ky Fan [2], and others.

In the theorems we will lean heavily on properties of solutions of a limiting autonomous system

$$
y^{\prime}=F(y) \text {. }
$$

A nondegenerate periodic solution of (2) will mean a periodic solution that does not correspond to a critical point of (2). That is, its graph in the plane is a simple closed curve.

The hypotheses of the following theorem are satisfied by numerous special cases and an example of one special case is given as a corollary to the theorem.

THEOREM 1. Let $f(t, z)$ and $F(z)$ be continuous in a region, $R$, of the plane and let $f(t, z) \rightarrow F(z)$ in $R$ as $t \rightarrow \infty$. Suppose that $f(t, z)$ is Lipschitzian with respect to $z$, that (2) has a unique solution through each point of $R$, that these solutions are all periodic and that the critical points of (2) are isolated. Also, suppose that all trajectories of (1) cross the nondegenerate periodic trajectories of (2) from inside to outside. Then every bounded solution of (1) which remains in $R$ is asymptotically almost periodic.

Proof. Let $x=x(t)$ be a bounded solution of (1) and let $\epsilon>0$ be given. Consider any sequence of reals

$$
\tau_{1}<\tau_{2}<\tau_{3}<\cdots \rightarrow \infty \text {. }
$$

Since $x(t)$ is bounded, for some subsequence

$$
t_{1}<t_{2}<t_{3}<\cdots \rightarrow \infty
$$

of (3) the sequence $\left\{x\left(t_{i}\right)\right\}$ converges.

Let

$$
x\left(t_{i}\right) \rightarrow p
$$

Case 1. $p$ is not a critical point of (2). Let the simple closed curve $C$ be the solution curve of (2) through $p$. For each $i$, the solution of (2) through $x\left(t_{i}\right)$ is a simple closed curve, $C_{i}$, interior to $C$ (we ignore the accidental cases where $x\left(t_{i}\right)$ may be a critical point of (2)). In fact, since solutions of (1) cross solutions of (2) from inside to outside, $C_{i}$ is interior to $C_{j}$ for $i<j$. 
Let $T$ be the period of the solution of (2) with initial condition $p$. Since $f(t, z) \rightarrow F(z)$ and since $f(t, z)$ is Lipschitzian, corresponding to any $\eta>0$ there exists $\xi>0$ such that if $q$ is a point of $C$ and $y(t)$ is the solution of (2) such that $y(a)=q$, then $\rho(x(a), y(a))<\xi$ implies $\rho(x(t), y(t))<\eta$ for $a \leqq t \leqq a+2 T$ (cf. $[1$, p. 105]). We will refer to this as continuity in the initial condition.

Using continuity in the initial condition and the period, $T$, of $y(t)$ we see that corresponding to $\epsilon / 2$ there exists a $\delta>0(\delta<\epsilon / 2)$ such that

$$
\rho(x(t), y(t))<\delta
$$

implies

$$
\rho(x(t+T), y(t+T))=\rho(x(t+T), y(t))<\epsilon / 2 .
$$

Hence, since $\delta<\epsilon / 2$, we have

$$
\rho(x(t), x(t+T))<\epsilon
$$

subject to there being a $y(t)$ satisfying (4).

Consider the $\delta$ neighborhood of $C$. That is, all points of the plane closer than $\delta$ to some point of $C$. We only need the inner annulus. Since $x\left(t_{i}\right) \rightarrow p$, by continuity of initial conditions, some $C_{i}$ is in the inner annulus. Because $x(t)$ crosses solutions of (2) from inside to outside, $x(t)$ is in the annulus for all $t>t_{i}$. That is, if $t^{*}>t_{i}$, there is some point $q$ of $C$ closer to the point $x\left(t^{*}\right)$ than $\delta$. Let $y(t)$ be the solution of (2) such that $y\left(t^{*}\right)=q$. Then, (4) is satisfied for this $t=t^{*}$ hence (5) is satisfied for this $t=t^{*}$. Since $t^{*}$ was arbitrary except for the condition $t^{*}>t_{i}$, the proof of this case is complete.

Case 2. $p$ is a critical point of (2). It is clear from the proof of Case 1 that if any point of the $\omega$-limit set of the orbit of $x(t)$ is not a critical point, then the corresponding solution of (2) is the entire $\omega$-limit set of the orbit. Thus, in Case 2, we may assume that all of the $\omega$-limit set of the orbit of $x(t)$ consists of critical points. These must be finite in number because $x(t)$ is bounded and the critical points are isolated.

The $\omega$-limit set consists of exactly one critical point. For if it consists of the $h>1$ points

$$
p_{1}, p_{2}, \cdots, p_{h}
$$

then let $\alpha$ be the minimum distance between these points and consider the $\alpha / 3$-neighborhoods of them. Select any infinite sequence of points $x\left(s_{i}\right)$ such that (a) $s_{1}<s_{2}<s_{3}<\cdots \rightarrow \infty$ and (b) for each $i$, $x\left(s_{i}\right)$ is in the complement of the set sum of the $\alpha / 3$-neighborhoods of $p_{1}, p_{2}, p_{3}, \cdots, p_{h}$. Since $x(t)$ is bounded, the points $\left\{x\left(s_{i}\right)\right\}$ have a convergent subsequence which is an $\omega$-limit point distinct from $p_{1}, p_{2}, p_{3}, \cdots, p_{h}$. 
Thus, in Case 2, there is a single $\omega$-limit point $p$ and $x(t) \rightarrow p$ as $t \rightarrow \infty$.

Trivially,

$$
\rho(x(t), x(t+T))<\epsilon
$$

for any $T>0$ and any $t$ sufficiently large (depending only on $\epsilon$ ). This completes the proof of the theorem.

In the theorem, one may replace "all trajectories of (1) cross those of (2) from inside to outside" by "all trajectories of (1) cross those of (2) from outside to inside" to secure a valid theorem. The proof is only altered in that the $C_{i}$ close down on $C$ instead of swelling out to $C$.

The following corollary is only one of many possible applications of Theorem 1. It is easily verified that the hypotheses of the theorem are implied by those of the corollary.

Corollary. For the system of equations

$$
x_{1}^{\prime}=F_{1}\left(x_{1}, x_{2}\right), \quad x_{2}^{\prime}=f\left(t, x_{1}, x_{2}\right),
$$

and

$$
x_{1}^{\prime}=F_{1}\left(x_{1}, x_{2}\right), \quad x_{2}^{\prime}=F_{2}\left(x_{1}, x_{2}\right),
$$

suppose that (7) has a unique solution through each point, that these solutions are all periodic and that the critical points are isolated. Assume $f(t, u, v) \rightarrow F_{2}(u, v)$ as $t \rightarrow \infty, v F_{1}(u, v) \geqq 0, u F_{2}(u, v) \leqq 0, f(t, u, v)$ $>F_{2}(u, v)$ if $v>0$ and $f(t, u, v)<F_{2}(u, v)$ if $v<0$. Then every bounded solution of (6) is asymptotically almost periodic.

For example, taking (6) to be

$$
x_{1}^{\prime}=x_{2}, \quad x_{2}^{\prime}=\left(1-\frac{x_{1} x_{2} a(t)}{1+x_{1}^{2} x_{2}^{2}}\right)\left(-x_{1}\right)
$$

where $1>a(t)>0, a(t) \rightarrow 0$ as $t \rightarrow \infty$, gives a system satisfying the conditions of the corollary.

The condition that the trajectories of (1) cross those of (2) consistently is not satisfied by an example of L. Markus [3] which, in rectangular coordinates, is the system

$$
\begin{aligned}
& x^{\prime}=-y+\cos t(\sin (\log t) / t), \\
& y^{\prime}=x+\sin t(\sin (\log t) / t), \quad t>0 .
\end{aligned}
$$

having as a solution 


$$
\begin{aligned}
& x=(2-\cos \log t) \cos t, \\
& y=(2-\cos \log t) \sin t, \quad t>0 .
\end{aligned}
$$

This solution is asymptotically almost periodic and illustrates the following theorem which is an easy consequence of continuity in the initial condition $[1$, p. 105].

Theorem 2. Suppose that in a region of the plane the continuous function $f(t, z)$ converges to the continuous function $F(z)$ as $t \rightarrow \infty$, that $f(t, z)$ is Lipschitzian with respect to $z$, that (2) has a unique solution through each point and that all of these solutions have the same period, T. Then, every bounded solution of (1) valid for all large $t$ is asymptotically almost periodic.

\section{REFERENCES}

1. Garrett Birkhoff and G.-C. Rota, Ordinary differential equations, Ginn, Boston, Mass., 1962.

2. $\mathrm{Ky}$ Fan, Les fonctions asymptotiquement presque-périodiques d'une variable entière et leur application d l étude de l'itération des transformations continues, Math. $Z$. 48 (1943), 685-711.

3. L. Markus, Asymptotically autonomous differential systems. Contributions to the theory of nonlinear oscillations, Vol. III, Annals of Mathematics Studies, No. 36, Princeton Univ. Press, Princeton, N. J., 1956.

4. J. S. W. Wong and T. A. Burton, Some properties of solutions of $u^{\prime \prime}(t)$ $+a(t) f(u) g\left(u^{\prime}\right)=0$. II, Monatsh. Math. 69 (1965), 368-374.

UNIVERSITY OF MISSOURI AND

UNIVERSITY OF IOWA 